\title{
Persistent Homology and Partial Similarity of Shapes
}

\author{
Barbara Di Fabio ${ }^{\mathrm{a}}$, Claudia Landi ${ }^{\mathrm{b}, \mathrm{a}}$ \\ ${ }^{a}$ University of Bologna, ARCES, Bologna, Italy \\ ${ }^{b}$ University of Modena and Reggio Emilia, DiSMI, Reggio Emilia, Italy
}

\begin{abstract}
The ability to perform shape retrieval based not only on full similarity, but also partial similarity is a key property for any content-based search engine. We prove that persistence diagrams can reveal a partial similarity between two shapes by showing a common subset of points. This can be explained using the MayerVietoris formulas that we develop for ordinary, relative and extended persistent homology. An experiment outlines the potential of persistence diagrams as shape descriptors in retrieval tasks based on both full and partial similarity.
\end{abstract}

Keywords: Mayer-Vietoris formula, extended persistence, Hausdorff distance 2010 MSC: 55N35, 68T10

\section{Introduction}

Distinguishing and recognizing deformable shapes is an important problem, encountered in numerous pattern recognition, computer vision and computer graphics applications. A major problem in the analysis of non-rigid shapes is finding similarity of deformable shapes which have only partial similarity, i.e., have similar as well as dissimilar parts.

Persistent homology is an algebraic tool for measuring topological features of spaces and functions. It allows for a multi-scale analysis of topological data. The scale at which a feature is significant is measured by its persistence. Motivated by the problem of describing and recognizing deformable shapes, persistence of 0-homology, also known as a size function, has been studied for years first in computer vision (Frosini, 1992; Verri et al., 1993) and later in computer graphics (Biasotti et al., 2006). Persistence of higher homology was originally introduced to

Email addresses: difabio@dm.unibo.it (Barbara Di Fabio), clandi@unimore.it (Claudia Landi) 
study alpha-shapes and later applied to pattern recognition (Carlsson et al., 2005). Using persistent homology, we obtain a shape descriptor in terms of a multiset of points of the plane, called a persistence diagram (or a barcode). Comparison of persistence diagrams by a distance such as the Hausdorff distance gives a stable methodology to assess shape similarity (Cohen-Steiner et al., 2007).

In this paper we study how the problem of assessing partial similarity can be dealt with using persistent homology.

To this end, we begin looking for Mayer-Vietoris formulas for persistence. A Mayer-Vietoris formula is a formula relating the ranks of the homology groups of spaces $X, A, B, C$ when $X=A \cup B$ and $C=A \cap B$. A Mayer-Vietoris formula for ordinary persistence has been obtained in (Di Fabio and Landi, 2010). Similar formulas also for relative and extended persistence are a novel contribution of this paper.

In ordinary persistence, the Mayer-Vietoris formula yields relationships among the persistence diagrams of $X, A, B, C$. These relationships indicate that the presence of $A$ in $X$ can be revealed by the presence of a common subset of points in the persistence diagrams of $A$ and $X$ (analogously for $B$ and $X$ ).

This idea is effectively used in the final experiment, dealing with the detection of both full and partial similarity of shapes.

\section{Background on ordinary, extended and relative persistence}

Persistence involves analyzing a topological space $Y$ through the evolution of a family of its subspaces nested by inclusion, i.e. a filtration. Applying homology to the filtration allows us to measure the lifetime of topological features in passing from a set of the filtration into a larger one. The basic assumption is that the longer a feature survives, the more meaningful or coarse the feature is for shape description. Vice-versa, noise and shape details are characterized by a shorter life.

A special type of filtration is the one formed using the sublevel sets $Y_{u}=$ $\varphi^{-1}((-\infty, u]), u \in \mathbb{R}$, of some real-valued function $\varphi: Y \rightarrow \mathbb{R}$.

The filtration $\left\{Y_{u}\right\}_{u \in \mathbb{R}}$ is used to define ordinary persistence as follows. Given $u \leq v \in \mathbb{R}$, we consider the inclusion of $Y_{u}$ into $Y_{v}$. This inclusion induces a homomorphism of homology groups $H_{k}\left(Y_{u}\right) \rightarrow H_{k}\left(Y_{v}\right)$ for every $k \in \mathbb{Z}$. Its image consists of the classes that live at least from $H_{k}\left(Y_{u}\right)$ to $H_{k}\left(Y_{v}\right)$ and is called the (ordinary) kth persistent homology group of $Y$ at $(u, v)$. When this group is finitely generated, we denote its rank by $\operatorname{Ord}_{k}^{u, v}(Y)$.

The ordinary persistence paradigm allows us to rank topological features with bounded lifetime by importance, according to the length of their life. Neverthe- 
less, it does not take into account the fact that some topological features could give rise to essential homology classes of $Y$ that never die along the filtration, still being produced by noise or expressing shape details. To overcome this problem, we can consider the following extension of persistence, in which all homology classes eventually die. This allows us to rank also topological features with unbounded lifetime.

To define extended persistence, also the superlevel sets $Y^{u}=\varphi^{-1}([u,+\infty))$, $u \in \mathbb{R}$, are used. First, we consider the filtration $\left\{\left(Y, Y^{u}\right)\right\}_{u \in \mathbb{R}}$, with $\mathbb{R}$ inversely ordered, in which the pair of sets $\left(Y, Y^{u}\right)$ is nested in $\left(Y, Y^{v}\right)$ whenever $u \geq v$. Given $u \geq v \in \mathbb{R}$, the inclusion of the pair $\left(Y, Y^{u}\right)$ into $\left(Y, Y^{v}\right)$ induces a homomorphism of homology groups $H_{k}\left(Y, Y^{u}\right) \rightarrow H_{k}\left(Y, Y^{v}\right)$ for every $k \in \mathbb{Z}$. Its image is called the relative kth persistent homology group of $Y$ at $(u, v)$. When this group is finitely generated, we denote its rank by $\operatorname{Rel}_{k}^{u, v}(Y)$. Finally, concatenating the two previous filtrations, we can define extended persistence as follows. Given $u, v \in \mathbb{R}$, the inclusion of the pair $\left(Y_{u}, \emptyset\right)$ into $\left(Y, Y^{v}\right)$ induces a homomorphism of homology groups $H_{k}\left(Y_{u}\right) \rightarrow H_{k}\left(Y, Y^{v}\right)$ for every $k \in \mathbb{Z}$. Its image is called the extended $k t h$ persistent homology group of $Y$ at $(u, v)$. When this group is finitely generated, we denote its rank by $\operatorname{Ext}_{k}^{u, v}(Y)$.

More details on ordinary, relative and extended persistence can be found in (Cohen-Steiner et al., 2009).

\section{Mayer-Vietoris formulas for persistent homology}

In this section we give Mayer-Vietoris formulas for ordinary, relative and extended persistent homology.

Given a triad $(X, A, B)$ with $X=A \cup B$, a Mayer-Vietoris formula is a relationship among the ranks of the homology groups of $X, A, B$ and $C=A \cap B$. It is obtainable from the Mayer-Vietoris sequence

$$
\cdots \rightarrow H_{k+1}(X) \rightarrow H_{k}(C) \rightarrow H_{k}(A) \oplus H_{k}(B) \rightarrow H_{k}(X) \rightarrow \cdots,
$$

when this sequence is exact. We recall that the homomorphism $H_{k+1}(X) \rightarrow H_{k}(C)$ maps $[z]$ to $\left[\partial\left(z_{\mid}\right)\right]$, the homomorphism $H_{k}(C) \rightarrow H_{k}(A) \oplus H_{k}(B)$ maps $[z]$ to $([z],[-z])$, and the homomorphism $H_{k}(A) \oplus H_{k}(B) \rightarrow H_{k}(X)$ maps $\left([z],\left[z^{\prime}\right]\right)$ to $\left[z+z^{\prime}\right]$.

According to the homology theory we use, different assumptions on the triad guarantee that the Mayer-Vietoris sequence is exact. To keep these assumptions to a minimum we shall work with Čech homology. Indeed, in Čech homology, it is 
sufficient that triads are compact and homology coefficients are taken in a vector space. Moreover, for triangulable spaces, Čech and simplicial homology coincide (Eilenberg and Steenrod, 1952).

In the context of persistent homology we endow $X$ with a continuous function $\varphi$, and $A, B$ and $C$ with the respective restrictions of $\varphi$. We assume that $(X, A, B)$ is a compact triad, and that the homology groups of the sublevel and superlevel sets of $\varphi, \varphi_{\mid A}, \varphi_{\mid B}, \varphi_{\mid C}$ are finitely generated. Moreover, we take homology coefficients in a field, so that persistent homology groups are vector spaces. These notations and assumptions will be maintained throughout the paper.

The novel idea in obtaining Mayer-Vietoris formulas for persistent homology is that of interlacing Mayer-Vietoris sequences with long exact sequences containing the maps that define the persistent homology groups. In the case of ordinary persistence, which is defined by the homomorphism $H_{k}\left(X_{u}\right) \rightarrow H_{k}\left(X_{v}\right)$ induced by inclusion, we shall use the long exact sequence of the pair $\left(X_{v}, X_{u}\right)$, and the analogous ones for $A, B, C$. In the case of relative persistence, which is defined by the homomorphism $H_{k}\left(X, X^{u}\right) \rightarrow H_{k}\left(X, X^{v}\right)$ induced by inclusion, we shall use the long exact sequence of the triple $\left(X, X^{v}, X^{u}\right)$, and the analogous ones for $A, B, C$. Finally, in the case of extended persistence, which is defined by the homomorphism $H_{k}\left(X_{u}\right) \rightarrow H_{k}\left(X, X^{v}\right)$ induced by inclusion, we shall use the long exact sequence of the proper triad $\left(X, X_{u}, X^{v}\right)$, and the analogous ones for $A, B, C$.

\subsection{A Mayer-Vietoris formula for ordinary persistence}

Since $(X, A, B)$ is a compact triad and $\varphi$ is continuous, also $\left(X_{u}, A_{u}, B_{u}\right)$ is a compact triad for each $u \in \mathbb{R}$. Therefore, for each pair of values $u, v$ in $\mathbb{R}$, with $u \leq v$, and $k \in \mathbb{Z}$, we can consider the following diagram

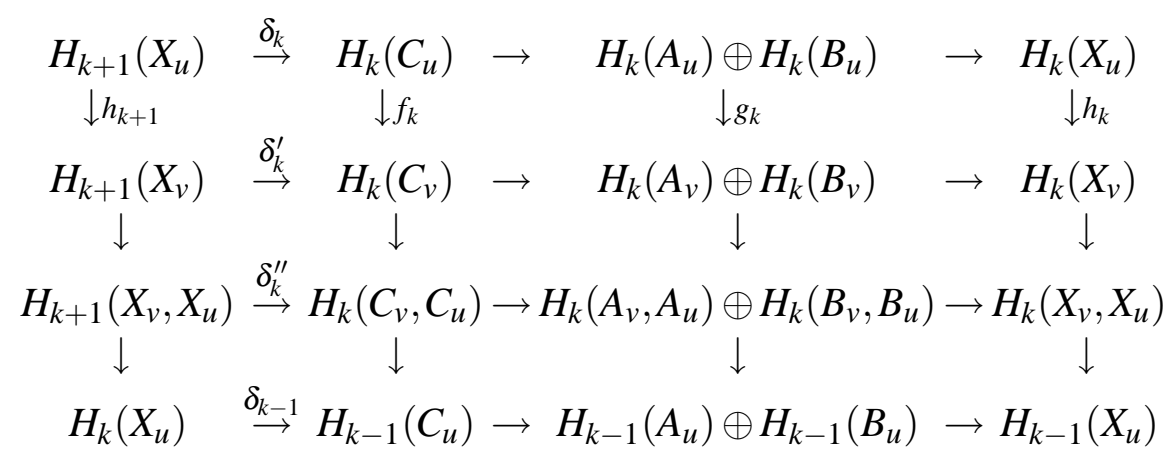

where the horizontal lines are part of exact Mayer-Vietoris sequences of the triads $\left(X_{u}, A_{u}, B_{u}\right),\left(X_{v}, A_{v}, B_{v}\right)$, and $\left(X_{v}, A_{v}, B_{v}\right)$ relative to $\left(X_{u}, A_{u}, B_{u}\right)$, and the vertical 
lines are part of long exact sequences of the pairs $\left(X_{v}, X_{u}\right),\left(A_{v}, A_{u}\right),\left(B_{v}, B_{u}\right)$, $\left(C_{v}, C_{u}\right)$.

Theorem 3.1. For every $u, v \in \mathbb{R}$, with $u \leq v$, and for every $k \in \mathbb{Z}$,

$$
\operatorname{Ord}_{k}^{u, v}(X)=\operatorname{Ord}_{k}^{u, v}(A)+\operatorname{Ord}_{k}^{u, v}(B)-\operatorname{Ord}_{k}^{u, v}(C)+\operatorname{rk} \delta_{k}^{\prime}-\operatorname{rk} \delta_{k}^{\prime \prime}+\operatorname{rk} \delta_{k-1},
$$

with $\delta_{k}^{\prime}, \delta_{k}^{\prime \prime}$, and $\delta_{k-1}$ as in Diagram (1).

This result has been proved in (Di Fabio and Landi, 2010). The proof is based on observing that $\operatorname{Ord}_{k}^{u, v}(X)=\operatorname{rkh}_{k}, \operatorname{Ord}_{k}^{u, v}(A)+\operatorname{Ord}_{k}^{u, v}(B)=\operatorname{rkg}_{k}, \operatorname{Ord}_{k}^{u, v}(C)=$ $\mathrm{rk} f_{k}$, with $h_{k}, g_{k}, f_{k}$ as in Diagram (1). Moreover, it uses some algebraic results that can be restated as follows.

Lemma 3.2. If $L_{k}, M_{k}, N_{k}$ are finitely generated vector spaces for every $k \in \mathbb{Z}$, and all the vertical and horizontal lines are exact in the diagram

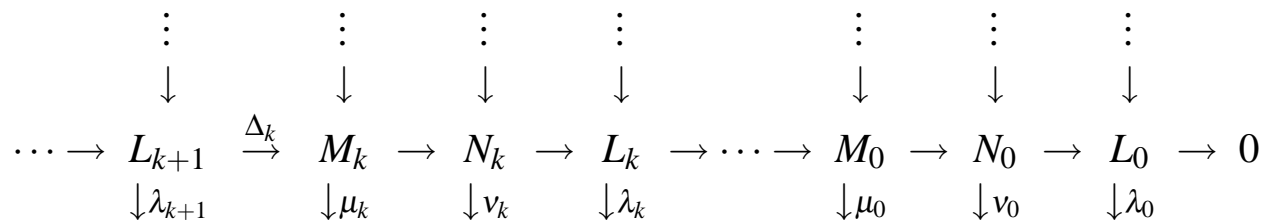

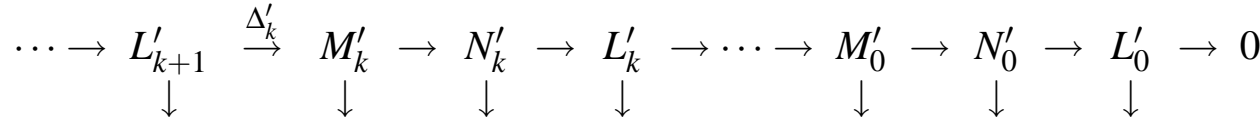

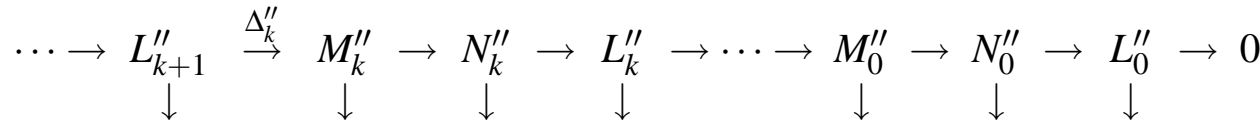

$$
\begin{aligned}
& \cdots \rightarrow \quad L_{k} \quad \stackrel{\Delta_{k-1}}{\rightarrow} M_{k-1} \rightarrow N_{k-1} \rightarrow L_{k-1} \rightarrow \cdots \rightarrow 0 \quad 0 \quad 0
\end{aligned}
$$

then $\mathrm{rk} \lambda_{k}=\mathrm{rk} v_{k}-\mathrm{rk} \mu_{k}+\mathrm{rk} \Delta_{k}^{\prime}-\mathrm{rk} \Delta_{k}^{\prime \prime}+\mathrm{rk} \Delta_{k-1}$.

\subsection{A Mayer-Vietoris formula for relative persistence}

In almost the same way as for ordinary persistence, we can deduce a MayerVietoris formula for relative persistence.

For each $u \geq v \in \mathbb{R},\left(X, X^{v}, X^{u}\right)$ is a compact triad with $X^{u} \subseteq X^{v} \subseteq X$. So, we can consider the following long exact sequence of the triple:

$$
\ldots \rightarrow H_{k}\left(X^{v}, X^{u}\right) \rightarrow H_{k}\left(X, X^{u}\right) \rightarrow H_{k}\left(X, X^{v}\right) \rightarrow H_{k-1}\left(X^{v}, X^{u}\right) \rightarrow \ldots
$$


Analogous exact sequences exist for $A, B, C$. Therefore we have the following diagram for every $k \in \mathbb{Z}$ :

$$
\begin{aligned}
& \underset{H_{k+1}\left(X, X^{u}\right) \stackrel{\bar{\delta}_{k}}{\rightarrow}}{\rightarrow} \underset{\substack{h_{k+1} \\
H_{k}\left(C, C^{u}\right) \\
\downarrow \bar{f}_{k}}}{\rightarrow} \quad H_{k}\left(A, A^{u}\right) \underset{\bar{g}_{k}}{\oplus H_{k}\left(B, B^{u}\right)} \rightarrow \begin{array}{c}
H_{k}\left(X, X^{u}\right) \\
\downarrow \bar{h}_{k}
\end{array} \\
& H_{k+1}\left(X, X^{v}\right) \stackrel{\bar{\delta}_{k}^{\prime}}{\rightarrow} \quad H_{k}\left(C, C^{v}\right) \rightarrow \quad H_{k}\left(A, A^{v}\right) \underset{\downarrow}{\oplus} H_{k}\left(B, B^{v}\right) \quad \rightarrow \quad H_{k}\left(X, X^{v}\right) \\
& H_{k}\left(X^{v}, X^{u}\right) \stackrel{\bar{\delta}_{k-1}^{\prime \prime}}{\rightarrow} H_{k-1}\left(C^{v}, C^{u}\right) \rightarrow H_{k-1}\left(A^{v}, A^{u}\right) \oplus H_{k-1}\left(B^{v}, B^{u}\right) \rightarrow H_{k-1}\left(X^{v}, X^{u}\right) \\
& H_{k}\left(X, X^{u}\right) \stackrel{\bar{\delta}_{k-1}}{\rightarrow} H_{k-1}\left(C, C^{u}\right) \rightarrow H_{k-1}\left(A, A^{u}\right) \oplus H_{k-1}\left(B, B^{u}\right) \rightarrow H_{k-1}\left(X, X^{u}\right)
\end{aligned}
$$

where the horizontal lines are part of exact Mayer-Vietoris sequences of the triads $(X, A, B)$ relative to $\left(X^{u}, A^{u}, B^{u}\right),(X, A, B)$ relative to $\left(X^{v}, A^{v}, B^{v}\right)$, and $\left(X^{v}, A^{v}, B^{v}\right)$ relative to $\left(X^{u}, A^{u}, B^{u}\right)$, and the vertical lines are part of long exact sequences of the triples $\left(X, X^{v}, X^{u}\right),\left(A, A^{v}, A^{u}\right),\left(B, B^{v}, B^{u}\right),\left(C, C^{v}, C^{u}\right)$.

Applying Lemma 3.2 to Diagram (2), and observing that $\operatorname{Rel}_{k}^{u, v}(X)=\mathrm{rk} \bar{h}_{k}$, $\operatorname{Rel}_{k}^{u, v}(A)+\operatorname{Rel}_{k}^{u, v}(B)=\operatorname{rk}_{k}, \operatorname{Rel}_{k}^{u, v}(C)=\operatorname{rk} \bar{f}_{k}$, we deduce the following result.

Theorem 3.3. For every $u, v \in \mathbb{R}$, with $u \geq v$, and for every $k \in \mathbb{Z}$,

$$
\operatorname{Rel}_{k}^{u, v}(X)=\operatorname{Rel}_{k}^{u, v}(A)+\operatorname{Rel}_{k}^{u, v}(B)-\operatorname{Rel}_{k}^{u, v}(C)+\operatorname{rk} \bar{\delta}_{k}^{\prime}-\operatorname{rk} \bar{\delta}_{k-1}^{\prime \prime}+\operatorname{rk} \bar{\delta}_{k-1},
$$

with $\bar{\delta}_{k}^{\prime}, \bar{\delta}_{k-1}^{\prime \prime}$, and $\bar{\delta}_{k-1}$ as in Diagram (2).

\subsection{Mayer-Vietoris formulas for extended persistence}

We now deduce Mayer-Vietoris formulas for extended persistence. The main difference with the previous cases is that, here, both sublevel and superlevel sets

are involved, with $u, v$ varying in $\mathbb{R}$. So, it will be necessary to distinguish the two cases $u<v$ and $u \geq v$.

Theorem 3.4. For every $u, v \in \mathbb{R}$, and $k \in \mathbb{Z}$, the following statements hold:

(i) If $u<v$, then

$$
\operatorname{Ext}_{k}^{u, v}(X)=\operatorname{Ext}_{k}^{u, v}(A)+\operatorname{Ext}_{k}^{u, v}(B)-E x t_{k}^{u, v}(C)+\operatorname{rk} \hat{\delta}_{k}^{\prime}-\operatorname{rk} \hat{\delta}_{k}^{\prime \prime}+\operatorname{rk} \hat{\delta}_{k-1}
$$

with $\hat{\delta}_{k}^{\prime}, \hat{\delta}_{k}^{\prime \prime}$, and $\hat{\delta}_{k-1}$ as in Diagram (4). 
(ii) If $u \geq v$, then

$$
\operatorname{Ext}_{k}^{u, v}(X)=\operatorname{Ext}_{k}^{u, v}(A)+\operatorname{Ext}_{k}^{u, v}(B)-E x t_{k}^{u, v}(C)+\operatorname{rk} \widetilde{\delta}_{k}^{\prime}-\operatorname{rk} \widetilde{\delta}_{k-1}^{\prime \prime}+\mathrm{rk} \widetilde{\delta}_{k-1},
$$

with $\widetilde{\delta}_{k}^{\prime}, \widetilde{\delta}_{k-1}^{\prime \prime}$, and $\widetilde{\delta}_{k-1}$ as in Diagram (5).

Proof. Let us consider the triad $\left(X, X_{u}, X^{v}\right)$ for fixed $u, v \in \mathbb{R}$, and, for the sake of conciseness, let us denote $X_{u} \cup X^{v}$ simply by ${ }^{\cup} X_{u}^{v}$ and $X_{u} \cap X^{v}$ by $\cap X_{u}^{v}$.

Since this triad $\left(X, X_{u}, X^{v}\right)$ is compact, and hence proper, for every $u, v \in \mathbb{R}$, its homology sequence

$$
\left.\ldots \rightarrow H_{k}\left(X_{u}, \cap^{v}\right)_{u}\right) H_{k}\left(X, X^{v}\right) \rightarrow H_{k}\left(X,{ }^{\cup} X_{u}^{v}\right) \rightarrow H_{k-1}\left(X_{u},{ }^{\cap} X_{u}^{v}\right) \rightarrow \ldots
$$

is exact (Eilenberg and Steenrod, 1952). Let us consider separately the two cases $u<v$ and $u \geq v$.

(i) If $u<v$, then $\cap^{v} X_{u}^{v}=\emptyset$. So (3) becomes

$$
\ldots \rightarrow H_{k}\left(X_{u}\right) \rightarrow H_{k}\left(X, X^{v}\right) \rightarrow H_{k}\left(X,{ }^{\cup} X_{u}^{v}\right) \rightarrow H_{k-1}\left(X_{u}\right) \rightarrow \ldots
$$

Applying analogous arguments to $A, B, C$, for every $k \in \mathbb{Z}$, we can consider the following diagram

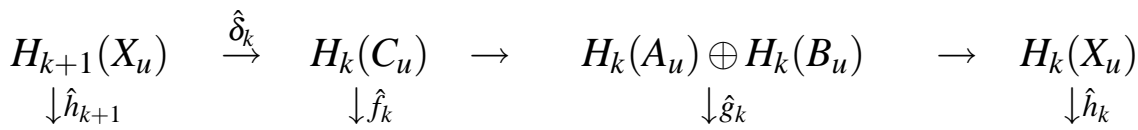

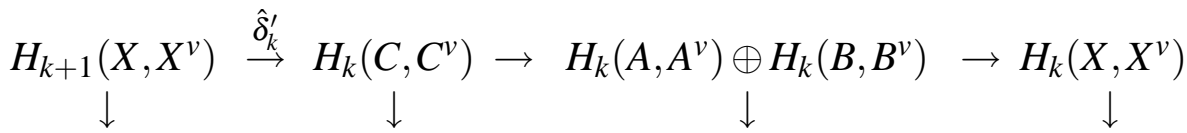

$$
\begin{aligned}
& H_{k+1}\left(X,{ }_{\downarrow} X_{u}^{v}\right) \stackrel{\hat{\delta}_{k}^{\prime \prime}}{\rightarrow} H_{k}\left(C,{ }_{\downarrow} C_{u}^{v}\right) \rightarrow H_{k}\left(A,{ }^{\cup} A_{u}^{v}\right) \underset{\downarrow}{\oplus} H_{k}\left(B,{ }^{\prime} B_{u}^{v}\right) \rightarrow H_{k}\left(X,{ }^{\cup} X_{u}^{v}\right) \\
& H_{k}\left(X_{u}\right) \stackrel{\hat{\delta}_{k-1}}{\rightarrow} H_{k-1}\left(C_{u}\right) \rightarrow H_{k-1}\left(A_{u}\right) \oplus H_{k-1}\left(B_{u}\right) \rightarrow H_{k-1}\left(X_{u}\right)
\end{aligned}
$$

where the horizontal lines are part of exact Mayer-Vietoris sequences of the triads $\left(X_{u}, A_{u}, B_{u}\right),(X, A, B)$ relative to $\left(X^{v}, A^{v}, B^{v}\right)$, and $(X, A, B)$ relative to $\left({ }^{\cup} X_{u}^{v}, \cup A_{u}^{v},{ }^{v} B_{u}^{v}\right)$, and the vertical lines are part of long exact sequences of the proper triads $\left(X, X_{u}, X^{v}\right)$, $\left(A, A_{u}, A^{v}\right),\left(B, B_{u}, B^{v}\right),\left(C, C_{u}, C^{v}\right)$.

We observe that $\operatorname{Ext}_{k}^{u, v}(X)=\operatorname{rk}_{k}, \operatorname{Ext}_{k}^{u, v}(A)+\operatorname{Ext}_{k}^{u, v}(B)=\operatorname{rkg}_{k}, \operatorname{Ext}_{k}^{u, v}(C)=$ $\mathrm{rk} \hat{f}_{k}$. Therefore, applying Lemma 3.2, we obtain the claimed formula.

(ii) If $u \geq v$, then ${ }^{v} X_{u}^{v}=X$. So (3) becomes

$$
\ldots \rightarrow H_{k}\left(X_{u},{ }^{\cap} X_{u}^{v}\right) \rightarrow H_{k}\left(X, X^{v}\right) \rightarrow H_{k}(X, X) \rightarrow H_{k-1}\left(X_{v},{ }^{\cap} X_{u}^{v}\right) \rightarrow \ldots
$$


This implies that, for every $k \in \mathbb{Z}, H_{k}\left(X_{u},{ }^{\cap} X_{u}^{v}\right)$ is isomorphic to $H_{k}\left(X, X^{v}\right)$. Consequently, by the commutativity of the diagram

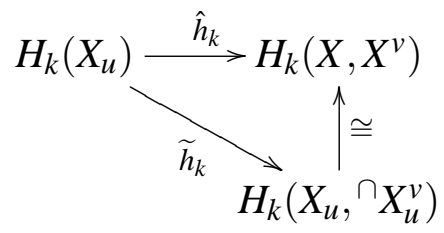

it follows that $\operatorname{Ext}_{k}^{u, v}(X)=\mathrm{rk} \widetilde{h}_{k}$. Similar considerations apply to $A, B, C$. Hence, for every $k \in \mathbb{Z}, E x t_{k}^{u, v}(A)+E x t_{k}^{u, v}(B)=\operatorname{rk} \widetilde{g}_{k}$ and $E x t_{k}^{u, v}(C)=\operatorname{rk} \widetilde{f}_{k}$, with $\widetilde{g}_{k}$ and $\widetilde{f}_{k}$ as in the following diagram

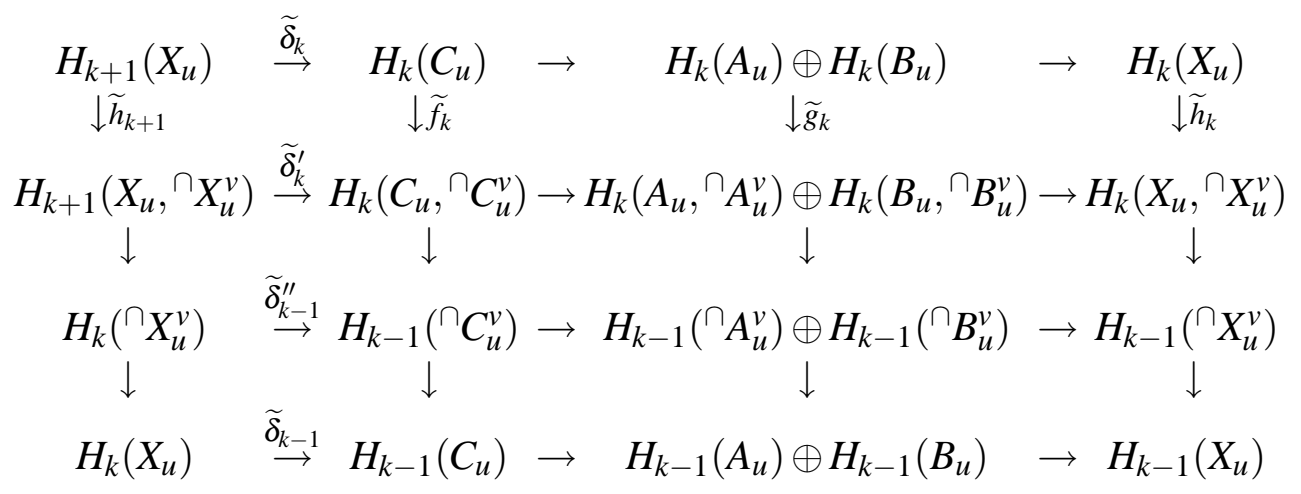

where the horizontal lines are part of exact Mayer-Vietoris sequences of the triads $\left(X_{u}, A_{u}, B_{u}\right),\left(X_{u}, A_{u}, B_{u}\right)$ relative to $\left(\cap X_{u}^{v}, \cap A_{u}^{v}, \cap B_{u}^{v}\right)$, and the triad $\left(\cap X_{u}^{v}, \cap A_{u}^{v}, \cap B_{u}^{v}\right)$, respectively, and the vertical lines are part of long exact sequences of the pairs $\left(X_{u}, \cap X_{u}^{v}\right),\left(A_{u}, \cap A_{u}^{v}\right),\left(B_{u}, \cap B_{u}^{v}\right),\left(C_{u}, \cap C_{u}^{v}\right)$.

The claim is again an immediate consequence of Lemma 3.2.

\section{Detection of sub-part similarity of shapes by persistence}

Persistent homology groups describe the shape of a space $Y$ as seen through a function $\varphi$, but the quantitative comparison of two shapes is performed using a representation of these groups, the so-called persistence diagram.

A persistence diagram $D_{k}(Y)$ is a set of points $(u, v) \in \mathbb{R}^{2}$ each corresponding to a pairing between the birth of a $k$-homology class at $u$ and its death at $v$ along the filtration of $Y$, union the points on the diagonal. $D_{k}(Y)$ can be partitioned into the ordinary sub-diagram, $D_{k}^{\text {Ord }}(Y)$, the relative sub-diagram, $D_{k}^{R e l}(Y)$, and the extended sub-diagram, $D_{k}^{E x t}(Y)$ (Cohen-Steiner et al., 2009). 
Algebraically, points of $D_{k}^{\text {Ord }}(Y)$ are those points $(u, v) \in \mathbb{R}^{2}$, with $u<v$, that have positive multiplicity $\mu_{k}^{u, v}(Y)$, where $\mu_{k}^{u, v}(Y)$ is equal to

$$
\lim _{\varepsilon \rightarrow 0^{+}}\left(\operatorname{Ord}_{k}^{u+\varepsilon, v-\varepsilon}(Y)-\operatorname{Ord}_{k}^{u-\varepsilon, v-\varepsilon}(Y)-\operatorname{Ord}_{k}^{u+\varepsilon, v+\varepsilon}(Y)+\operatorname{Ord}_{k}^{u-\varepsilon, v+\varepsilon}(Y)\right) .
$$

Ordinary persistence can reveal sub-part similarity of shapes by showing common subsets of points in the corresponding persistence diagrams. This fact is illustrated by an example in Table 1, and implicitly stated in the following result from (Di Fabio and Landi, 2010).

Theorem 4.1. For every $p=(u, v) \in \mathbb{R}^{2}$, with $u<v$, and every $k \in \mathbb{Z}$, the following statements hold:

(i) Denoting $\delta_{k}^{\prime \prime}: H_{k+1}\left(X_{v}, X_{u}\right) \rightarrow H_{k}\left(C_{v}, C_{u}\right)$ by $\delta_{k}^{v, u}$, if $p \in D_{k}^{\text {Ord }}(X) \cup D_{k}^{\text {Ord }}(C)$ and $\lim _{\varepsilon \rightarrow 0^{+}}\left(\operatorname{rk} \delta_{k}^{v-\varepsilon, u-\varepsilon}-\operatorname{rk} \delta_{k}^{v-\varepsilon, u+\varepsilon}+\operatorname{rk} \delta_{k}^{v+\varepsilon, u+\varepsilon}-\operatorname{rk} \delta_{k}^{v+\varepsilon, u-\varepsilon}\right) \leq 0$, then $p \in D_{k}^{\text {Ord }}(A) \cup D_{k}^{\text {Ord }}(B)$.

(ii) Assuming that both $\varphi$ and $\varphi_{\mid C}$ have at most a finite number of homological $k$-critical values, if $p \in D_{k}^{\text {Ord }}(A) \cup D_{k}^{\text {Ord }}(B)$ and at least one of its coordinates is neither a homological $(k+1)$-critical value of $\varphi$ nor a homological $(k-1)$-critical value of $\varphi_{\mid C}$, then $p \in D_{k}^{\text {Ord }}(X) \cup D_{k}^{\text {Ord }}(C)$.

The proof of this result is based on the Mayer-Vietoris formula for ordinary persistence given in Theorem 3.1, which yields a relation linking the multiplicities of points of $D_{k}^{\text {Ord }}(X), D_{k}^{\text {Ord }}(A), D_{k}^{\text {Ord }}(B)$, and $D_{k}^{\text {Ord }}(C)$.

\section{Experiment and discussion}

In Table 2, we show a retrieval test on a data set containing 15 shapes: 5 humans, 5 horses and 5 centaurs (Bronstein et al., 2008). Each shape differs by an articulation and additional parts. We assume as ground truth that a man and a centaur are dissimilar in the sense of a full similarity criterion, yet, parts of these shapes (the upper part of the centaur and the upper part of the man) are similar. Likewise, a horse and a centaur are similar because they share a common part (bottom part of the horse body). At the same time, a man and a horse are dissimilar.

Each shape is modeled by taking as topological space the set of black pixels with the 8-neighbor adjacency topology, and as filtering function the function "minus the distance from the center of the bounding box". Shapes are analyzed 

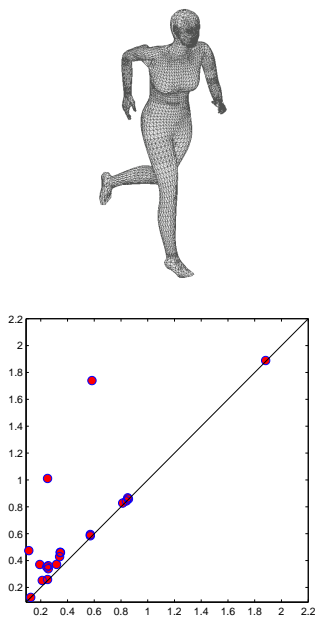

$D_{1}^{\text {Ord }}(X)$
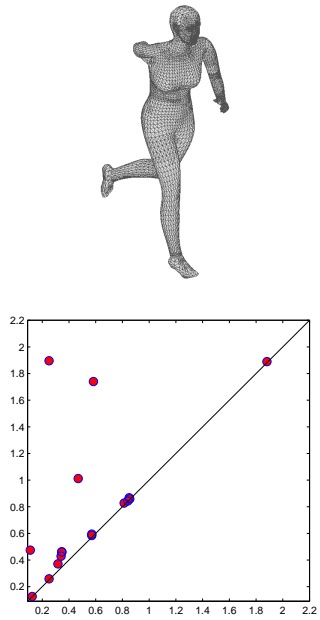

$D_{1}^{\text {Ord }}(A)$
$\Pi$

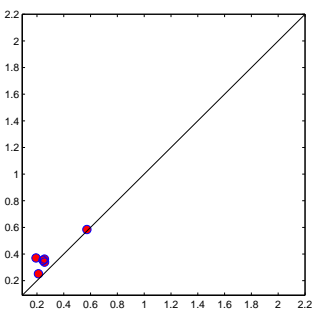

$D_{1}^{\text {Ord }}(B)$

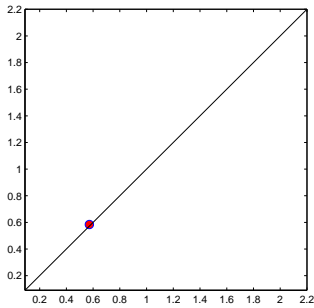

$D_{1}^{\text {Ord }}(C)$

Table 1: The 1-homology ordinary persistence diagrams of a triangle mesh $X$ and its sub-parts $A, B, C$ are computed with respect to the function $\varphi(x, y, z)=|y+z|+|y-z|$.

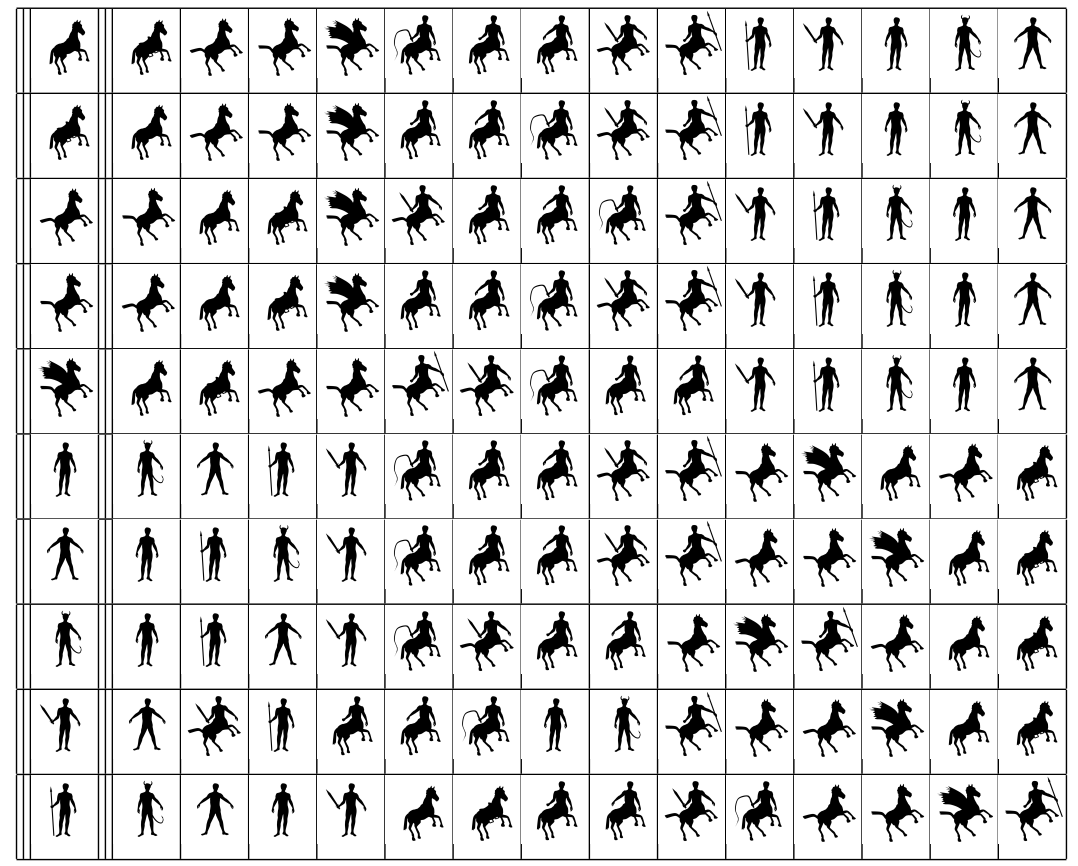

Table 2: Retrieval from the mythological 2D dataset. The first column contains the query shapes. In each row retrieved shapes are ordered by increasing distance. The first retrieved shape is omitted being always equal to the query shape. 
using only ordinary and extended 0 -th persistence. Table 2 shows the retrieval obtained using the Hausdorff distance on extended sub-diagrams and the partial Hausdorff pseudo-distance on the ordinary sub-diagrams. The partial Hausdorff pseudo-distance automatically selects a fixed fraction of the persistence diagrams points giving the best matching and uses only this subset to compute the Hausdorff distance (Huttenlocher et al., 1993). We considered the $98 \%$ of points of each ordinary persistence sub-diagram.

Our test shows that, by using persistent homology to retrieve shapes from this dataset, the similarity relations assumed as ground truth are respected.

Based on these results we conclude that persistent homology performs nicely for the needs of automatic partial similarity assessment, and could be exploited in content-based shape search engines.

Since in this paper we developed Mayer-Vietoris formulas also for relative and extended persistence, possible advances could concern obtaining from these formulas results analogous to Theorem 4.1. One difficulty in this respect is that one should first develop an appropriate notion of multiplicity for points to belong to these sub-diagrams.

\section{References}

Biasotti, S., Giorgi, D., Spagnuolo, M., Falcidieno, B., 2006. Size functions for 3d shape retrieval, in: SGP '06: Proceedings of the fourth Eurographics symposium on Geometry processing, pp. 239-242.

Bronstein, A.M., Bronstein, M.M., Bruckstein, A.M., Kimmel, R., 2008. Analysis of two-dimensional non-rigid shapes. Intl. Journal of Computer Vision , 67-88.

Carlsson, G., Zomorodian, A., Collins, A., Guibas, L., 2005. Persistence barcodes for shapes. International Journal of Shape Modeling 11, 149-187.

Cohen-Steiner, D., Edelsbrunner, H., Harer, J., 2007. Stability of persistence diagrams. Discrete Comput. Geom. 37, 103-120.

Cohen-Steiner, D., Edelsbrunner, H., Harer, J., 2009. Extending persistence using Poincaré and Lefschetz duality. Foundations of Computational Mathematics 9, 133-134.

Di Fabio, B., Landi, C., 2010. A Mayer-Vietoris formula for persistent homology with an application to shape recognition in the presence of occlusions. Technical report 2806. Università di Bologna. Http://amsacta.cib.unibo.it/2806/. 
Eilenberg, S., Steenrod, N., 1952. Foundations of algebraic topology. Princeton University Press, Princeton, New Jersey.

Frosini, P., 1992. Measuring shapes by size functions, in: D. P. Casasent (Ed.), Society of Photo-Optical Instrumentation Engineers (SPIE) Conference Series, pp. 122-133.

Huttenlocher, D.P., Klanderman, G.A., Rucklidge, W., 1993. Comparing images using the Hausdorff distance. IEEE Trans. Pattern Anal. Mach. Intell. 15, 850863.

Verri, A., Uras, C., Frosini, P., Ferri, M., 1993. On the use of size functions for shape analysis. Biol. Cybern. 70, 99-107. 